\title{
Assessing the merits of existing pancreatic cancer biomarkers
}

\author{
Paweł Kiczmer, Alicja Prawdzic Seńkowska, Błażej Szydło, Elżbieta Świętochowska, \\ Zofia Ostrowska
}

Pancreatic ductal adenocarcinoma (PDAC) suffers from a very poor prognosis because early stages of the disease are asymptomatic and thus diagnosis is delayed until late. Discovering a suitable PDAC biomarker could thereby improve PDAC treatment by having an early diagnosis. The carbohydrate antigen, CA 19-9, currently used for diagnostics, may help in assessing the disease stage, however it is unsuitable for screening purposes. PDAC specific nucleotides can be detected in plasma but not at the early stages of the cancer. Furthermore, measuring circulating tumour cells (CTCs) in patient blood entails high costs and is only useful for advanced stage disease. Other potential PDAC marker candidates are Laminin $\gamma 2 \mathrm{~A}, \mathrm{Cyclophilin} \mathrm{B}$ and blood circulating adipokines, which seem to hold particular promise. At present, making early PDAC diagnosis is limited. The potential markers described herein might in the future be introduced into clinical practice however further studies are still required. Using combinations of several biomarkers also merit consideration, which may increase the overall sensitivity and specificity of PDAC detection.

NOWOTWORY J Oncol 2017; 67, 3: 201-205

Key words: PDAC, biomarkers, miRNA, adipokine, adiponectin, laminin, cyclophilin

\section{Introduction}

Pancreatic ductal adenocarcinoma (PDAC) represents about $3 \%$ of all human malignant tumours [1]. It is one of those cancers occurring in highly developed countries, especially for men in their 70 s and 80 s. Over $20 \%$ cases are diagnosed when carcinogenesis is already at an advanced stage, including infiltration to adjacent organs or the presence of distant metastases. At the beginning of the disease, progression is asymptomatic whereas in late stage, the clinical picture becomes abruptly aggravated with symptoms depending on tumour localization, size and progression stage. When suspecting pancreatic cancer, ultrasonography is recommended, whose sensitivity is estimated to be at $80-90 \%$ [2]. Other medical tests applied are computed tomography and endoscopic retrograde cholangiopancreatography. Morbidity rates are very high; the five-year survival rate for radically treated patients being $3-18 \%$ [2]. Radical surgery is possible in only $20 \%$ cases due to the advanced stage of cancer upon diagnosis [3, 4]. The ability for detecting this cancer early could thus considerably improve its prognosis. Discovering a new biological marker may thereby accelerate the diagnosis and enable the delivery of appropriate treatment at an early disease stage. The presented work attempts to evaluate the utility of several potential pancreatic cancer markers (Tab. I).

\section{CA 19-9, CA 125, CA 242, CEA}

Carbohydrate antigens CA 19-9, CA 125 and CA 242 are often investigated and described, together with carcinoembryonic antigen (CEA), so that specificity and sensitivity may be improved when these biomarkers are jointly measured. CA 19-9 is a sialylated Lewis (a) antigen which is absent in $3-7 \%$ of the general population with a Lewis $(a-b)$ bloodtype antigen [5].

Amidst all the known PDAC markers, only CA 19-9 has been currently employed in diagnostics. Its sensitivity rate is $79 \%$ and specificity $82 \%$, which limits its clinical value making it impossible to distinguish pancreatic cancer from benign pancreatic diseases and multiple carcinoma [6]. Because CA 19-9 is not tumour-specific protein, serum concentrations may also be elevated in patients with inflammatory diseases of the digestive tract and liver disease; particularly cholelithiasis [7]. 
Table I. Clinical relevance of potential PDAC biomarkers

\begin{tabular}{ll}
\hline Potential screening markers & Potential monitoring markers \\
\hline Nucleotides & CA $19-9$ \\
Adipokines & CA 125 \\
Laminin 2a & CEA \\
Cyclophilin B & CA 242 \\
& CTC \\
\hline
\end{tabular}

In the 1980s in Japan, an attempt to introduce CA 19-9 for screening purposes was made [8]. A study on 70,940 healthy individuals showed increased concentrations (> $37 \mathrm{U} / \mathrm{mL}$ ) of the marker in 1063 cases (1.5\%). There was no evidence of cancer in patients having concentrations of 37-100 U/mL whereas CA 19-9 levels were $>100 \mathrm{U} / \mathrm{mL}$ in 89 patients in whom 15 cancer cases (19\%) were detected which included 4 instances of PDAC; two of these qualifying for radical surgery. This study thereby concluded that CA 19-9 as a PDAC screening marker was unsuitable. Nevertheless, CA 19-9 is the marker of choice when monitoring patients with ductal carcinoma of the pancreas [7]. High postoperative CA 19-9 levels have been associated with poor survival and may identify those patients who, as an alternative, should receive systemic therapy [6]. Dong et al. reported that a preoperative serum CA 19-9 concentration > $338.45 \mathrm{IU} / \mathrm{mL}$ may be a prognostic factor for poor prognosis [9]. According to Hartwig et al., postoperative CA 19-9 decreases are associated with longer survival whereas increased post-operative CA 19-9 might in addition be a prognostic factor for a poorer prognosis [3]. Some researchers suggest that postoperative CA 19-9 elevation is associated with a microscopic positive tumour margin and poorer survival as a consequence [10].

CA 19-9 could also be useful in monitoring the pharmacotherapy of PDAC. Boone et al. observed that a $>50 \%$ decrease in CA 19-9 levels is correlated with a longer survival of patients who underwent neoadjuvant therapy [11]; Tzeng et al. obtained similar outcomes [12]. Further studies are needed to confirm whether failure to normalise CA 19-9 levels could be an independent predictor of shorter survival.

CA 125 or MUC16 belong to the mucin family of glycoproteins [13] and is mostly known for detecting ovarian cancer [14]. To date, it has not been suggested for PDAC screening, nonetheless it may play a complementary role in PDAC diagnosis and prognosis besides that of CA 19-9 [15]. Chinese Study Group for Pancreatic Cancer considered CA 125 to be useful in the diagnosis and detection of metastasis, as well as in selecting the appropriate therapy and for monitoring disease progression, regression and recurrence $[16,17]$. Interestingly, according to Luo et al. CA 125 is superior to CA 19-9 for predicting the resectability of pancreatic cancer where high levels may indicate an unresectable pancreatic cancer [18].
CEA (carcino-embryonic antigen) is glycoprotein involved in cell adhesion and has been used for diagnosing recurrent colorectal cancer [19] and it might also prove useful for detecting PDAC. As a single marker, CEA is of low sensitivity (30-88\%) but when combined with others sensitivity may become increased [20].

CA 242 is also a tumour marker for sialylated Lewis carbohydrates that has been suggested for PDAC diagnosis. It appears to have the highest specificity when compared to CA 19-9, CA 125 and CEA but has a low sensitivity (67.8\%), however it may improve the CA 19-9 discrimination rate [21-23].

A valuable study by Yu-Lei et al. jointly measured serum levels of CA 19-9, CEA, CA 125 and CA 242 in PDAC patients and achieved a higher detectability of $90.4 \%$ sensitivity and $93.8 \%$ specificity, than when markers were measured alone [21].

\section{Nucleotides}

MiRNA molecules are small single stranded RNAs consisting of 18-22 nucleotides, which modulate posttranscriptional expression of several genes. MiRNA deregulation may not only be associated with upregulation of genes involved in cancer progression (cell proliferation, migration and invasion), but also with apoptosis evasion, and chemoresistance [24]. A study by Xu et al. suggested that miR-486-5p allows the discrimination of patients between PDAC, chronic pancreatitis and normal subjects (AUC $=0.861$ ) [25]. The study demonstrated that miR-938 is comparable to CA 19-9 when differentiating patients with PDAC from CP.

Because the miRNA profile consists of a panel of up-regulated or down-regulated miRNAs responsible for many of the aforementioned aspects of tumorigenesis, then evaluating this profile may provide a more sensitive and more specific method than measuring a single miRNA. For instance, miR-16 and miR-196a together with CA19-9, constitute an effective set of first stage tumour markers. Indeed, some workers believe that miRNAs may have a role in assessing a patient's prognosis and their selection for treatment [26]. As an example, upon measuring circulating non-coding RNAs (ncRNAs) in cases of advanced PDAC, useful information can be gained, such as predicting the patient's response to chemotherapy. Furthermore, Humeau et al. reported that PDAC-specific MiRNA can be detected in saliva samples [27] and indicate that MiR-23a and MiR-23b are both present in patients with intraductal papillary $\mathrm{mu}$ cinous neoplasms; otherwise known as non-invasive PDAC precursors. Another suggested and promising PDAC biomarker is circulating cell-free DNA (cfDNA). This arose from the conception of a 'liquid biopsy', which consist of analysing pieces of nucleic acids from tumour cells in collected blood sample $[28,29]$. CfDNA is derived from somatic DNA released into the systemic circulation following cellular necrosis and 
apoptosis. Hadano et al. found that KRAS-mutated cfDNA was associated with significantly poorer survival in patients with resectable tumours [29] and it was suspected that the cfDNA tumour found may correlate with micrometastases, which are undetectable by imaging methods. This suggests that cfDNA-positive patients may be considered for any preoperative options such as neo-adjuvant chemotherapy. These outcomes have been confirmed by other studies [28, $30,31]$. Kisiel et al. detected PDAC hypermetylated DNA in pancreatic juice [32] and reported that such PDAC specific DNA-methylation markers may discriminate between early disease stage from normal tissue. Matsubayashi et al. likewise detected hypermethylated PDAC cfDNA in pancreatic juice [33]. In summary, both cfDNA and MiRNA should be therefore considered as potential PDAC screening markers. It is also worth mentioning that MiRNA can be isolated from the saliva, which is a non-invasive method allowing premalignant pancreatic lesions to become detected.

\section{Circulating tumour cells (CTCs)}

CTCs are tumour cells circulating in blood vessels with metastatic potential. They have been detected in patients suffering from breast, lung and prostate cancers [34]. Kulemann et al. found CTCs in $73 \%$ of PDAC patients $(n=11)$; with CTCs being found in patients at every stage of the disease [34]. Earl et al. measured CTCs in $20 \%$ of such cases $(n=35)$ [31] with six out of all seven patients with CTCs having metastases; one patient had a resectable tumour. These outcomes lead us to conclude that CTC may be a sensitive PDAC biomarker. In addition, PDAC is poorly vascularised and its invasion is often limited to the liver, and only involves other organs at very advanced stages [31, 35, 36].

Bissolati et al. investigated CTCs in portal blood collected during PDAC surgery [35] and showed no differences in survival rate between CTC-positive and CTC-negative patients. An increased risk of liver metastasis was however found in CTC-positive patients. It should be noted that CTC isolation is very expensive and we believe that it cannot be used as a screening biomarker but may be applied when monitoring PDAC progression.

\section{Adipokines}

Adiponcetin is a 244-aminoacids cell signalling peptide (cytokine) synthesised in adipocytes with serum concentrations being inversely proportional to the percentage of body fat [37]. Adiponectin plasma levels correlate inversely with cancer risk; especially those associated with obesity and insulin resistance. This cytokine has been suggested as a potent inhibitor of angiogenesis in vivo [38]. Other studies also indicate that, in vitro, it may inhibit endothelial cell migration and induce cell apoptosis via activation of caspases. Concentrations of plasma adiponectin is significantly higher in PDAC patients $(24.95 \mathrm{mg} / \mathrm{mL}$ ) compared to healthy $(10.4 \mathrm{mg} / \mathrm{mL})$ patients and chronic pancreatitis cases (10.3 mg/mL) [37]; Chang et al. obtained similar results [39]. In contrast, Pezilli et al. observed no significant differences in adiponectin serum levels between controls and a PDAC group [40]. There is evidence that the adiponectin serum level is inversely proportional to PDAC risk [41]. Serum adipokine levels increase during the course of PDAC. Based on the aforementioned studies, adiponectin may thus become a future PDAC biomarker.

Another described adipokine, leptin, is a 167 aminoacid protein where its serum concentrations are proportional to the body fat mass [37]. Leptin is supposed to be a marker differentiating autoimmune pancreatitis patients from those with chronic pancreatitis (CP) and pancreatic cancer where significantly lower serum levels were measured in patients with CP and PDAC compared to autoimmune pancreatitis [40]; without any significant differences between CP and PDAC [40]. Sakamoto et. al also observed no differences in serum leptin concentrations between PDAC and CP groups [42]. In summary, adiponectin may play a part as a PDAC biomarker, whereas leptin has low significance in the diagnosis of this cancer.

\section{Laminin}

Laminins are high molecular weight glycoproteins contributing to basement membrane structure. They are made up of three polypeptide chains: the $\alpha, \beta$ and $\gamma$ encoded collectively into 11 genetic variants [43]. The gamma chain emerges in three different genetic polymorphisms; one of which encodes subtype laminin gamma 2 (LAMC2) which has been suggested as a potential PDAC biomarker [44]. Kosanam et al. measured increased serum levels in PDAC patients $(382.2 \mathrm{ng} / \mathrm{mL}$ ) compared to those for benign tumours $(140 \mathrm{ng} / \mathrm{mL})$ and healthy individuals $(87 \mathrm{ng} / \mathrm{mL})$. LamC2 (AUC $=0.87$ ) outperforms CA19-9 (AUC = 0.82) in differentiating between healthy and cancer patients. In contrast, CA19-9 is superior upon comparing benign and PDAC patients. The combined use of LAMC2 and CA19.9 could thereby permit a more effective discrimination between age-matched normal and benign patients from PDAC patients than the single marker measurement of CA 19.9 [44].

\section{Cyclophilin B}

Cyclophilins are group of proteins acting as molecular chaperones that fold, translocate and process newly synthesized proteins. Cyclophilin B (СурB) is a $21-k D a$ protein belonging to the cyclophilin family of peptidyl-prolyl cis-trans isomerases. By reversible modification of their protein structure, cyclophilins also serve as signalling switches, regulating the activities of transforming growth factor $\beta$ receptor, epidermal growth factor receptor, tyrosine kinases and transcription factors such as c-Myb and interferon regulatory factor 4 [45]. Cyclophilins are widely expressed in the human body and highly conserved throughout evolution. 
Increased expression of cyclophilin B was observed in stomach and breast cancers [46]. Ray et al. measured increased Cyp B serum levels in 24 patients with PDAC [47]. The mean CypB level was $295 \mathrm{ng} / \mathrm{mL}$ in the PDAC group and $60 \mathrm{ng} / \mathrm{mL}$ in the controls $(n=24)$. The authors believe that increased secretion of CypB into the bloodstream may be caused by oxidative stress in the hypoxic tumour environment.

\section{Conclusion}

At present, there are no tests available for confirming PDAC that are easy-to-perform, specific, sensitive, non-invasive and that are of prognostic value. Early detection and choosing appropriate treatment may significantly reduce mortality and increase survival time in patients suffering from pancreatic cancer. Although advanced methods such as computed tomography and ultrasonography are commonly used in medical practice, the mortality rate remains very high. The main reason is that diagnosis is made too late. Twenty percent cases are diagnosed at a very advanced stage and qualified only for surgical treatment. Thirty percent of such patients undergoing surgery are expected to relapse. The only appropriate marker available, CA 19-19, is however unsuitable for asymptomatic cases. At this time, hopes can be pinned on measuring adipokines as in the aforementioned instance of adiponectin and MiRNA profiles should also be considered as useful PDAC biomarkers. Measuring CTCS however is expensive and labour-intensive and therefore inappropriate for clinical practice. It is thus our opinion that a combination of measuring several biomarkers might increase the sensitivity and specificity of detection and allow such means to be introduced for the screening of PDAC.

\section{Conflicts of interest: none declared}

\author{
Alicja Prawdzic Seńkowska \\ Department of Medical and Molecular Biology \\ Medical University of Silesia \\ 19 Jordana St. \\ 41-808 Zabrze, Poland \\ e-mail: alicja.senkowska@gmail.com
}

Received: 24 Mar 2017

Accepted: 15 May 2017

\section{References}

1. Krzakowski M, Warzocha K et al. (eds.). Zalecenia postępowania diagnostyczno-terapeutycznego wnowotworach złośliwych 2013 rok: praca zbiorowa. Gdańsk: Via Medica, 2013: 140-150.

2. Kordek R, Jassem J, Jeziorski A et al. (eds.). Onkologia: podręcznik dla studentów i lekarzy. Gdańsk: Via Medica, 2013: 198-202.

3. Hartwig W, Vollmer CM, Fingerhut A et al. Extended pancreatectomy in pancreatic ductal adenocarcinoma: definition and consensus of the International Study Group for Pancreatic Surgery (ISGPS). Surgery 2014; 156: 1-14.

4. Tamburrino $D$, Partelli $S$, Crippa $S$ et al. Selection criteria in resectable pancreatic cancer: a biological and morphological approach. World J Gastroenterol 2014; 20: 11210-11215.

5. Goonetilleke KS, Siriwardena AK. Systematic review of carbohydrate antigen (CA 19-9) as a biochemical marker in the diagnosis of pancreatic cancer. Eur J Surg Oncol 2007; 33: 266-270.
6. Duffy MJ, Sturgeon C, Lamerz R et al. Tumour markers in pancreatic cancer: a European Group on Tumour Markers (EGTM) status report. Ann Oncol 2010; 21: 441-447.

7. Galli C, Basso D, Plebani M. CA 19-9: handle with care. Clin Chem Lab Med 2013; 51: 1369-1383.

8. Kim JE, Lee KT, Lee JK et al. Clinical usefulness of carbohydrate antigen 19-9 as a screening test for pancreatic cancer in an asymptomatic population. J Gastroenterol Hepatol 2004; 19: 182-186.

9. Dong $\mathrm{Q}$, Yang $\mathrm{XH}$, Zhang $\mathrm{Y}$ et al. Elevated serum CA19-9 level is a promising predictor for poor prognosis in patients with resectable pancreatic ductal adenocarcinoma: a pilot study. World J Surg Oncol 2014; 12: 171.

10. Hata S, Sakamoto Y, Yamamoto Y et al. Prognostic impact of postoperative serum CA 19-9 levels in patients with resectable pancreatic cancer. Ann Surg Oncol 2012; 19: 636-641.

11. Boone BA, Steve J, Zenati MS et al. Serum CA 19-9 response to neoadjuvant therapy is associated with outcome in pancreatic adenocarcinoma. Ann Surg Oncol 2014; 21: 4351-4358.

12. Tzeng CW, Balachandran A, Ahmad M et al. Serum carbohydrate antigen 19-9 represents a marker of response to neoadjuvant therapy in patients with borderline resectable pancreatic cancer. HPB (Oxford) 2014; 16: 430-438.

13. Duraisamy S, Ramasamy S, Kharbanda S et al. Distinct evolution of the human carcinoma-associated transmembrane mucins, MUC1, MUC4 AND MUC16. Gene 2006; 373: 28-34.

14. Suh KS, Park SW, Castro A et al. Ovarian cancer biomarkers for molecular biosensors and translational medicine. Expert Rev Mol Diagn 2010; 10 : 1069-1083.

15. Coppin L, Benomar K, Corfiotti F et al. CA-125, but not galectin-3, complements CA 19-9 for discriminating ductal adenocarcinoma versus non-malignant pancreatic diseases. Pancreatology 2016; 16: 115-120.

16. Liu L, Xiang J, Chen R et al. The clinical utility of CA125/MUC16 in pancreatic cancer: a consensus of diagnostic, prognostic and predictive updates by the Chinese Study Group for Pancreatic Cancer (CSPAC). Int J Oncol 2016; 48: 900-907.

17. Liu L, Xu HX, Wang WQ et al. Serum CA125 is a novel predictive marker for pancreatic cancer metastasis and correlates with the metastasis-associated burden. Oncotarget 2016; 7: 5943-5956.

18. Luo G, Xiao Z, Long J et al. CA125 is superior to CA19-9 in predicting the resectability of pancreatic cancer. J Gastrointest Surg 2013; 17: 2092-2098.

19. Nicholson BD, Shinkins B, Pathiraja I et al. Blood CEA levels for detecting recurrent colorectal cancer. Cochrane Database Syst Rev 2015(12): CD011334.

20. Ballehaninna UK, Chamberlain RS. Biomarkers for pancreatic cancer: promising new markers and options beyond CA 19-9. Tumour Biol 2013; 34: 3279-3292.

21. Gu YL, Lan C, Pei H et al. Applicative value of serum CA19-9, CEA, CA125 and CA242 in diagnosis and prognosis for patients with pancreatic cancer treated by concurrent chemoradiotherapy. Asian Pac J Cancer Prev 2015; 16: 6569-6573.

22. Ozkan H, Kaya M, Cengiz A. Comparison of tumor marker CA 242 with CA 19-9 and carcinoembryonic antigen (CEA) in pancreatic cancer. Hepatogastroenterology 2003; 50: 1669-1674.

23. Zhang Y, Yang J, Li H et al. Tumor markers CA19-9, CA242 and CEA in the diagnosis of pancreatic cancer: a meta-analysis. Int $J$ Clin Exp Med 2015; 8: 11683-11691.

24. Huang J, Liu J, Chen-Xiao K et al. Advance in microRNA as a potential biomarker for early detection of pancreatic cancer. Biomark Res 2016; 4: 20. eCollection 2016.

25. Xu J, Cao Z, Liu W et al. Plasma miRNAs effectively distinguish patients with pancreatic cancer from controls: a multicenter study. Ann Surg 2016; 263: 1173-1179.

26. Previdi MC, Carotenuto P, Zito D et al. Noncoding RNAs as novel biomarkers in pancreatic cancer: what do we know? Future Oncol 2017; 13: 443-453.

27. Humeau M, Vignolle-Vidoni A, Sicard F et al. Salivary microRNA in pancreatic cancer patients. PLoS One 2015; 10: e0130996.

28. Takai E, Totoki $Y$, Nakamura $\mathrm{H}$ et al. Clinical utility of circulating tumour DNA for molecular assessment and precision medicine in pancreatic cancer. Adv Exp Med Biol 2016; 924: 13-17.

29. Hadano N, Murakami Y, Uemura K et al. Prognostic value of circulating tumour DNA in patients undergoing curative resection for pancreatic cancer. Br J Cancer 2016; 115: 59-65.

30. Bettegowda C, Sausen M, Leary RJ et al. Detection of circulating tumour DNA in early- and late-stage human malignancies. Sci Transl Med 2014; 6: 224ra24. 
31. Earl J, Garcia-Nieto S, Martinez-Avila JC et al. Circulating tumour cells (Ctc) and kras mutant circulating free Dna (cfdna) detection in peripheral blood as biomarkers in patients diagnosed with exocrine pancreatic cancer. BMC Cancer 2015; 15: 797.

32. Hu C, Hart SN, Bamlet WR et al. Prevalence of pathogenic mutations in cancer predisposition genes among pancreatic cancer patients. Cancer Epidemiol Biomarkers Prev 2016; 25: 207-211.

33. Matsubayashi $\mathrm{H}$, Canto $\mathrm{M}$, Sato $\mathrm{N}$ et al. DNA methylation alterations in the pancreatic juice of patients with suspected pancreatic disease. Cancer Res 2006; 66: 1208-1217.

34. Kulemann B, Pitman MB, Liss AS et al. Circulating tumour cells found in patients with localized and advanced pancreatic cancer. Pancreas 2015; 44: 547-550.

35. Bissolati M, Sandri MT, Burtulo G et al. Portal vein-circulating tumour cells predict liver metastases in patients with resectable pancreatic cancer. Tumour Biol 2015; 36: 991-996.

36. Hess KR, Varadhachary GR, Taylor SH et al. Metastatic patterns in adenocarcinoma. Cancer 2006; 106: 1624-1633.

37. Dranka-Bojarowska D, Lekstan A, Olakowski M et al. The assessment of serum concentration of adiponectin, leptin and serum carbohydrate antigen-19.9 in patients with pancreatic cancer and chronic pancreatitis. J Physiol Pharmacol 2015; 66: 653-663.

38. Brakenhielm E, Veitonmaki N, Cao R et al. Adiponectin-induced antiangiogenesis and antitumour activity involve caspase-mediated endothelial cell apoptosis. Proc Natl Acad Sci U S A 2004; 101: 2476-2481.
39. Chang MC, Chang YT, Su TC et al. Adiponectin as a potential differential marker to distinguish pancreatic cancer and chronic pancreatitis. Pancreas 2007; 35: 16-21.

40. Pezzilli R, Barassi A, Corsi MM et al. Serum leptin, but not adiponectin and receptor for advanced glycation end products, is able to distinguish autoimmune pancreatitis from both chronic pancreatitis and pancreatic neoplasms. Scand J Gastroenterol 2010; 45: 93-99.

41. Bao Y, Giovannucci EL, Kraft $\mathrm{P}$ et al. A prospective study of plasma adiponectin and pancreatic cancer risk in five US cohorts. J Nat/ Cancer Inst 2013; 105: 95-103.

42. Sakamoto $H$, Kimura $H$, Sekijima $M$ et al. Plasma concentrations of angiogenesis-related molecules in patients with pancreatic cancer. Jpn J Clin Oncol 2012; 42: 105-112.

43. Aumailley M. The laminin family. Cell Adh Migr 2013; 7: 48-55.

44. Kosanam H, Prassas I, Chrystoja CC et al. Laminin, gamma 2 (LAMC2): a promising new putative pancreatic cancer biomarker identified by proteomic analysis of pancreatic adenocarcinoma tissues. Mol Cell Proteomics 2013; 12: 2820-2832.

45. Fang F, Flegler AJ, Du P et al. Expression of cyclophilin B is associated with malignant progression and regulation of genes implicated in the pathogenesis of breast cancer. Am J Pathol 2009; 174: 297-308.

46. Meng DQ, Li PL, Xie M. Expression and role of cyclophilin B in stomach cancer. Genet Mol Res 2015; 14: 5346-5354.

47. Ray P, Rialon-Guevara KL, Veras E et al. Comparing human pancreatic cell secretomes by in vitro aptamer selection identifies cyclophilin B as a candidate pancreatic cancer biomarker. J Clin Invest 2012; 122: 1734-1741. 